\title{
ASSISTÊNCIA DE ENFERMAGEM À PESSOA COM ACIDENTE VASCULAR CEREBRAL
}

\author{
Shirley Rangel Gomes ${ }^{1}$, Mônica Senna²
}

\begin{abstract}
RESUMO: Pesquisa bibliográfica na qual selecionou-se 32 resultados a partir dos 156 artigos e livros, localizados nas bases de dados: Medline, Lilacs. Scielo, Capes e busca manual. Objetiva-se analisar os conhecimentos descritos na literatura relacionados com cuidados de enfermagem a pacientes com pré-disposição e acometimento de acidente vascular cerebral, de modo a considerar as ações diárias de enfermagem que contribuem para o controle dos fatores iatrogênicos e reabilitação do sujeito. Em seguida, realizou-se a análise dos diferentes contextos da questão à luz do referencial teórico de Hinds, Chaves e Cypress de modo que os estudos encontrados foram integrados em subtemas, como a perspectiva de cada quadro conceptual. Os resultados foram descritos no total de cinco etapas, consolidando as ações de enfermagem pertinentes a cada um.
\end{abstract}

PALAVRAS-CHAVE: Cuidados de enfermagem; Acidente Vascular Cerebral; Atividades cotidianas.

\section{NURSING CARE TO THE SUBJECT WITH CEREBRAL VASCULAR ACCIDENT}

\begin{abstract}
Literature review, bringing together 32 results from literature search with 156 articles and books, located in the following databases: Medline, Lilacs. Scielo, Capes and manual search. It aims to examine literature-described knowledge on nursing care to patients with pre-provision and involvement of stroke in order to consider daily nursing actions contributing to the control of iatrogenic factors and subject's rehabilitation. It followed the analysis of the different contexts of the issue in the light of Hinds, Chaves and Cypress's theoretical background so that the studies were integrated into sub-topics such as the prospect of each conceptual framework. The results were described in a total of five stages, consolidating nursing actions relevant to each one of them.
\end{abstract}

KEYWORDS: Nursing care; Stroke; Activities of daily living.

\section{ASISTENCIADE ENFERMERÍA EN ELACCIDENTE VASCULAR CEREBRAL}

RESUMEN: Se trata de una revisión de la literatura, con 32 resultados de búsqueda bibliográfica en 156 artículos y libros, que se encuentran en bases de datos: Medline, Lilacs, Scielo, Capes, y búsqueda manual. Tiene por objeto examinar los conocimientos de la literatura relacionada a la atención de enfermería a pacientes con predisposición y la participación de un accidente cerebrovascular con el fin de analizar las acciones diarias de enfermería que contribuyen al control de los factores iatrogénicos y la rehabilitación del sujeto. Se realizó el análisis de los diferentes contextos de la cuestión a la luz de los teóricos de referencia Hinds, Chaves y Cypress, de modo que los estudios fueron integrados en temas secundarios, como la perspectiva de cada cuadro conceptual. Los resultados fueron descriptos en un total de cinco etapas, consolidando las acciones de enfermería correspondientes a cada una de ellas.

PALABRAS CLAVES: Atención de enfermería; Accidente cerebrovascular; Actividades cotidianas.

${ }^{1}$ Enfermeira do Centro de Reabilitação do Hospital Geral de Guarús. Mestranda em Enfermagem. Mestrado em Enfermagem Profissional Assistencial, Universidade Federal Fluminense-UFF. Especialização em MBA Gestão Estratégica de Hospitais, Fundação Getúlio Vargas-FGV-RJ.

${ }^{2}$ Assistente Social. Doutora em Saúde Pública. Fundação Oswaldo Cruz-FIOCRUZ.

Autor Correspondente:

Shirley Rangel Gomes

Av. Senador José Carlos Pereira Pinto, 400 - 28080-790 - Campos dos Goytacazes-RJ

Recebido: $12 / 11 / 07$

E-mail: gomeshira@gmail.com 


\section{INTRODUÇÃO}

Este artigo é parte de um trabalho de pesquisa do curso de mestrado, no qual estão sendo desenvolvidos processos investigatórios sobre assistência de enfermagem às pessoas em processo de reabilitação, acometidas por Acidente Vascular Cerebral (AVC), visando ampliar o conhecimento sobre o objeto, como estratégia retroalimentadora do ensino, da pesquisa e da assistência.

Considera-se relevante o estudo, visto que o AVC vem sendo constituído, na população brasileira, desde 1960, como causa principal de internações, mortalidade e disfuncionalidade, acometendo a faixa etária acima de 50 anos, superando, até mesmo, as doenças cardíacas e o câncer ${ }^{(1)}$.

No Brasil, ocorreram importantes transformações no seu padrão de morbidade e mortalidade, relacionadas, principalmente, às seguintes condições: a redução da mortalidade precoce, especialmente aquela relacionada a doenças infecciosas e parasitárias; o aumento da expectativa de vida ao nascer, com o conseqüente incremento da população idosa e das causas de adoecimento e morte mais prevalentes nesse grupo etário; e o processo acelerado de urbanização e de mudanças socioculturais que respondem, em grande parte, pelo aumento dos acidentes e das violências, bem como por determinadas mudanças no perfil epidemiológico de algumas doenças transmissíveis ${ }^{(2)}$.

O impacto das doenças crônicas sobre as sociedades humanas é crescente, especialmente, quando evolui para graus variados de incapacidade ou morte. Na sociedade atual encontra-se um grande número de indivíduos com alguma disfunção e esse número cresce diariamente no mundo. As causas e conseqüências da limitação são diversas e, especialmente, as conseqüências do viver com algum tipo de deficiência são influenciadas pelas condições gerais de vida e pelas políticas sociais e econômicas que são adotadas pelos Estados com vistas ao bem-estar de seus cidadãos. Ao adquirir uma lesão, a pessoa sofre limitações para exercer o controle sobre suas condições de vida (acesso à escola, ao trabalho, ao lazer, à satisfação de suas necessidades básicas, entre outros) ${ }^{(3)}$.

Neste artigo, objetiva-se analisar o conhecimento descrito na literatura relacionado à assistência de enfermagem ao paciente acometido de AVC, de maneira a considerar as ações cotidianas que contribuem para o controle de iatrogenias dos sujeitos.

\section{AÇÕES DO ENFERMEIRO E O PROCESSO DE REABILITAR}

Reabilitação, de acordo com o Programa Mundial da Organização das Nações Unidas (ONU) para Pessoas com Deficiência,

[...] é um processo de duração limitada e com o objetivo definido, com vista a permitir que uma pessoa com deficiência alcance o nível físico, mental e /ou social funcional ótimo, proporcionando-lhe assim os meios de modificar a sua própria vida. Pode compreender medidas com vista a compensar a perda de uma função ou uma limitação funcional, como ajudas técnicas e outras medidas para facilitar ajustes ou reajustes sociais ${ }^{(4: 35)}$.

A Reabilitação ainda pode ser compreendida como "um processo dinâmico, contínuo, progressivo e principalmente educativo, tendo como objetivos a restauração funcional do indivíduo, sua reintegração à família, a comunidade e à sociedade"(5), ou "um processo genuinamente dinâmico, orientado para a promoção da saúde e qualidade de vida do indivíduo enfermo ou incapacitado de alcançar seu maior nível possível de independência funcional física, mental, espiritual, social e econômica"(6).

Cabe ao enfermeiro da reabilitação: o desenvolvimento de um processo interacional e transdisciplinar que favoreça o planejamento; implementação e avaliação de medidas terapêuticas de enfermagem voltadas para a educação e promoção da saúde com enfoque no autocuidado ${ }^{(7)}$; proporcionar o envolvimento e a participação ativa e sistemática do cliente, família e pessoas significativas em relação aos cuidados a serem desempenhados em casa ${ }^{(8-10)}$.

\section{MATERIAL E MÉTODOS}

Este estudo constitui-se num recorte, reunindo 32 artigos, a partir de pesquisa bibliográfica com 156 artigos, localizados nas bases de dados: Medline, Lilacs, Scielo, Capes, realizada no período de agosto e setembro de 2006.

A seleção de fontes para o levantamento das publicações foi realizada por meio de busca em artigos localizados na base de dados, busca manual em livros e jornais científicos. Foram inclusos artigos que relacionavam a assistência de enfermagem, enfermagem baseada em evidência, acidente vascular cerebral e atividades cotidianas. Houve combinação de descritores: assistência de enfermagem e acidente vascular cerebral; acidente vascular cerebral e 
enfermagem baseada em evidência para possibilitar as buscas. Artigos de reabilitação que não estivessem relacionados a pessoas acometidas por AVC foram excluídos.

Em seguida, houve a análise dos diferentes contextos da temática à luz do referencial teórico de Hinds, Chaves e Cypress ${ }^{(11)}$ de modo que os estudos encontrados fossem integrados em subtemas, conforme a perspectiva conceitual de cada contexto.

\section{RESULTADOS}

Os resultados foram descritos em subtemas baseados no ciclo da doença descrito na literatura: fatores pré-disponentes, início dos sintomas da doença, atendimento do cliente nos níveis de saúde: primário, secundário e terciário, bem como o retorno dessa pessoa à condição de reabilitado( ${ }^{(2)}$. Concomitante à análise dos subtemas, incluíram-se as ações de enfermagem pertinentes a cada uma, baseadas nos achados da literatura.

\section{Etapas de adoecimento do paciente com acidente vascular cerebral $e$ as ações de enfermagem}

$\mathbf{1}^{\text {a }}$ Etapa: Promover acompanhamento e orientação às pessoas com hipertensão arterial e outras doenças cardiovasculares, diabetes mellitus e demais fatores de risco de AVC, bem como identificar o tempo de ocorrência dos primeiros sinais e sintomas do Acidente Vascular Encefálico Isquêmico e a assistência necessária, refletindo sobre os resultados de prevenção de seqüelas e de novos episódios.

Ações de enfermagem: Os enfermeiros da equipe do Programa Saúde da Família (PSF) que fazem acompanhamento das pessoas e os fatores de risco associados, promovem o diagnóstico de enfermagem, interação com os mesmos através de modelos assistenciais, prevenindo e reconhecendo os riscos ${ }^{(11-}$ 12). A atuação dos profissionais no controle da hipertensão e do tabagismo e a redução dos casos fatais devido ao tratamento rápido e especializado, associado ao crescente avanço nos manejos clínicos estão relacionadas ao declínio das mortes resultantes de Acidente Cerebral Encefálico ${ }^{(13)}$. O reconhecimento e ações são norteados por cartilhas elaboradas pelo Ministério da Saúde, que preconizam cuidados, especialmente, aos realizados no Sistema Único de Saúde ${ }^{(14)}$.

$2^{\text {a }}$ Etapa: Atendimento emergencial em
Serviço de Pronto Socorro, a fim de identificar os diagnósticos e iniciar terapêutica medicamentosa, bem como ações de enfermagem que visem minimizar os riscos, seqüelas, deformidades e iatrogenias.

Ações de enfermagem: $O$ atendimento e reconhecimento dos diagnósticos para AVC, proporcionam a grande diferença no resultado do tratamento. O paciente atendido, diagnosticado clinicamente, tomograficamente e tratado com anticoagulantes nas três primeiras horas após o início do acometimento, tem maior chance de minimizar as seqüelas decorrentes do $\mathrm{AVC}^{(9-10)}$. $\mathrm{O}$ atendimento, feito pela equipe interdisciplinar, possibilita um rápido diagnóstico e tratamento adequados. É nesta fase que o enfermeiro inicia a avaliação do cliente, diagnosticando os fatores de risco para iatrogenias, desde o momento da consulta até seu tratamento hospitalar. Prevenir as seqüelas inerentes à doença e atentar-se para o período do doente no hospital deve ser compreendido como um grande diferencial no processo de reabilitação da pessoa. A Sistematização de Assistência de Enfermagem pode ser baseada em escalas de avaliação neurológica, com identificação dos déficits motores e sensoriais que dão indícios para o local de AVC, ${ }^{(15)}$ bem como utilização de escalas na avaliação neurológica do paciente com Acidente Vascular Encefálico (AVE) isquêmico do Nathional Institute of Healt(16), e Escala de Medida de Independência Funcional ${ }^{(17-18)}$ são úteis, para acompanhar o curso da doença e determinar o prognóstico, as ações preventivas de iatrogenias, bem como reabilitadoras.

$3^{\text {a }}$ Etapa: Internação do paciente e a assistência do cliente na clínica de internação: assistência interdisciplinar com vistas a desenvolver ações que promovam a reabilitação das seqüelas, prevenção de iatrogenias, preparo do cliente e cuidador para alta hospitalar.

Ações de enfermagem: A internação do cliente, em muitos aspectos, é difícil de ser realizada de maneira rápida e efetiva, em virtude da sobrecarga do Sistema Único de Saúde, das Unidades Hospitalares que não dispõem de recursos ou mesmos vagas para recebimento deste paciente. Logo, a escolha da clínica está diretamente ligada à disponibilidade de vagas. A escolha entre unidades especializadas em neurologia e as tradicionais com equipe interdisciplinar não tem sido considerada importante, pois os resultados da assistência não apresentam resultados significantes ${ }^{(19)}$. Essa fase de tratamento é vital para o prosseguimento 
e sucesso da reabilitação do cliente. A assistência direta, a orientação do cuidador $^{(20)}$, a relevância da identificação das dificuldades de cuidar do cliente em domicílio ${ }^{(21)}$, as ações desenvolvidas pelo enfermeiroprofessor e aluno no treinamento da família ${ }^{(22)}$, as implicações relacionadas ao perfil do sujeito que irá assumir e continuar, em domicílio, as ações terapêuticas ${ }^{(23)}$, avaliar os aspectos cognitivos da pessoa que continuará com os cuidados a fim de elaborar a melhor estratégia de orientação para o mesmo $^{(24)}$, buscar subsídios na prática baseada em evidência que possibilite nortear as ações, como, por exemplo, a questão nutricional importante para recuperação do cliente( ${ }^{(25)}$.

Conforme relatos na literatura, é importante estabelecer critérios para o atendimento deste cliente internado: disposição do quarto de uma pessoa com AVC - o lado hemiplégico deve ficar voltado para o centro do quarto, para favorecer a máxima estimulação dos sentidos ${ }^{(26)}$; medidas posturais e mobilização - quando um paciente está clinicamente estável e apresenta um bom nível de consciência, convém começar nas primeiras 24 a 48 horas com mobilização passiva e ativa na cama, controle da cabeça e do tronco e transferência para poltrona.

Dessa maneira, previnem-se as úlceras de pressão, diminui-se a incidência de trombose venosa e de complicações respiratórias, ao mesmo tempo em que se acelera a recuperação. Importante afirmar que as atividades devem ser realizadas pelo paciente, sempre que for possível; pela equipe de enfermagem; pelo familiar/cuidador devidamente orientado pelo enfermeiro ${ }^{(1,7)}$; eliminação - freqüentemente aparecem a impactação fecal e a incontinência urinária. Avaliar a necessidade de cateterismo vesical e atentar para integridade cutâneo-mucosa ${ }^{(9)}$; programar atividades físicas - essas atividades têm por objetivo melhorar o condicionamento físico geral, reduzir os fatores de risco do AVC e proporcionar vivência e experiência em atividades em grupo, favorecendo a reinserção social. Devem ser iniciados após avaliação em parceria com profissionais especializados.

Alimentação - a presença de disfagia pode provocar desidratação, e associa-se a infecções pulmonares, a uma maior mortalidade intra-hospitalar e a um pior prognóstico funcional. Nos primeiros dias após um AVC, enquanto se avalia a disfagia e se inicia a alimentação, recomenda-se manter hidratação venosa e evitar sondagem naso gástrica ou naso enteral, que dificulta a reabilitação. Se, após 7 a 8 dias, não conseguir um adequado aporte nutricional, está indicada a sondagem (sem que isso implique abandono do tratamento) ${ }^{(26)}$.

$4^{a}$ Etapa: Alta para o domicílio: um desafio para o enfermeiro, paciente e cuidador para a continuidade da assistência.

Ações de enfermagem: Em muitas unidades de saúde, o cuidador é preparado para dar continuidade ao tratamento domiciliar, por meio de estratégias educacionais, sobre condutas possíveis de serem realizadas que minimizem ou previnam iatrogenias. O cliente, quando transferido para o domicílio, pode desenvolver muitas complicações. Entre elas: deformidades do lado plégico, lesões cutâneas, broncoaspiração, distúrbios vesicointestinais, infecções urinárias, distúrbios dentários, isolamento social, entre outros.

Deve-se possibilitar ao cuidador familiar a utilização, no domicílio, de conhecimentos adquiridos durante a estada no hospital, o que deve ser realizado, com a orientação sistematizada, na qual o enfermeiro deve utilizar as suas habilidades de educador, conhecer o perfil do cuidador familiar, identificar facilidades e dificuldades para o cuidado domiciliar. Outra estratégia que possibilita a continuidade da assistência hospitalar baseia-se no Programa de Internação Domiciliar que promove a internação do cliente, com vínculo no hospital de origem e suporte para os clientes que atendam o perfil, definido pela resolução, para esse tipo de internação(27-31). A literatura aponta também a importância da criação de políticas públicas, sociais e de saúde direcionadas ao cuidado domiciliar e à cuidadora, em virtude de, na maioria das vezes, um elemento só atuar no cuidado o que ocasiona a sobrecarga física e mental ${ }^{(32)}$. É importante atentar-se para o fato de que não somente a pessoa vitimada de AVC, mas o seu cuidador também passa a ser cliente.

5 atapa: Inserção em Unidade de Reabilitação Especializada, com equipe interdisciplinar.

Ações de enfermagem: Considera-se esta unidade de atendimento um elo entre a Unidade Hospitalar e os Programas de Saúde Pública. Esta unidade de trabalho é atualmente um grande desafio de atendimento, não só pela carência de unidades especializadas, mas pela assistência de enfermagem estar ligada à assistência realizada desde a primeira etapa. O enfermeiro atua com cuidados, principalmente com a orientação familiar, nos seguintes aspectos: reabilitação motora - a reabilitação motora é dirigida para se conseguir o nível máximo de mobilidade e um 
alinhamento articular normalizado ${ }^{(25)}$; complicações na fase de reabilitação - atentar para espasticidade e dor no ombro hemiplégico ${ }^{(26)}$; atividades da vida diária - além de avaliar a capacidade de realizar ou não uma determinada tarefa, avalia-se a necessidade de supervisão ou assistência, o tempo gasto para realizar cada tarefa e a necessidade de adaptações e modificações no ambiente e ações do cuidador ${ }^{(27,32)}$; risco de novo infarto cerebral - É muito importante identificar as pessoas com risco de recorrência de AVC. Por meio de estudos epidemiológicos, sabe-se que cerca de 10 por cento das pessoas que sofreram um AVC têm risco de voltarem a apresentar novo AVC no primeiro ano após o evento. Após o primeiro ano, esse risco diminui ${ }^{(28)}$; preparo do indivíduo para viver com limitações impostas pelas seqüelas do AVC; promover a re-inserção do cliente, nos projetos de saúde pública, a fim de prevenir novos acidentes cerebrovasculares, por meio do sistema de Referência e Contra-Referência do SUS ${ }^{(31)}$.

É importante ressaltar que as ações preventivas não são exclusivas do nível primário. Devem-se estabelecer os cuidados e a assistência retroalimentadora do processo de cuidar, conforme resumido no Esquema 1.

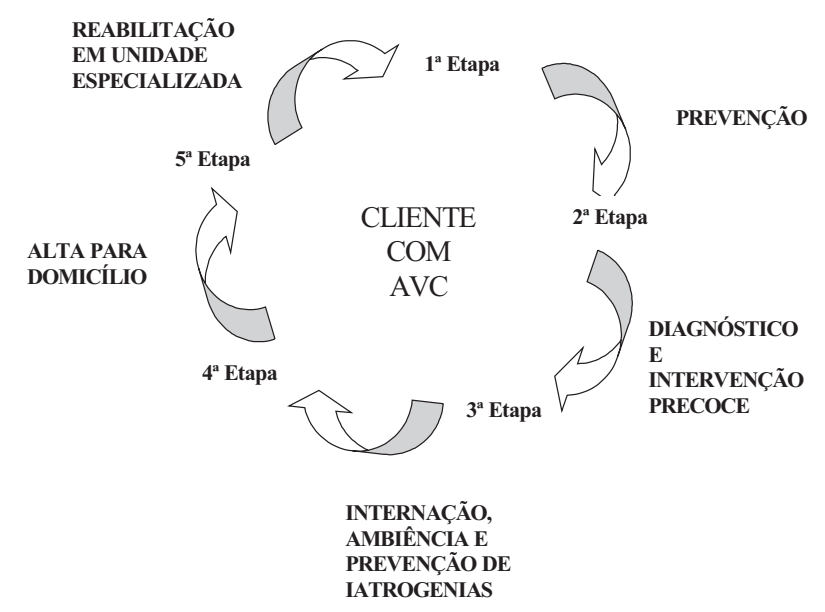

Esquema 1 - Sistematização da Assistência de Enfermagem e o processo de adoecimento da pessoa com AVC

\section{CONSIDERAÇÕES}

A análise da literatura descreve todas as ações pertinentes ao enfermeiro, norteando-o na sistematização da assistência em todas as etapas descritas como subtemas, desde as condições prédisponentes ao AVC bem como as intervenções objetivas em todo o processo de adoecimento.
Observa-se que as ações devem ser executadas em parceria entre as diversas equipes envolvidas na assistência, a fim de possibilitar ao cliente, a reabilitação das suas potencialidades, prevenção de novos acidentes, reconhecimento dos fatores de risco e ações específicas no tratamento de co-morbidades e doenças crônico-degenerativas.

Na unidade de reabilitação, o enfermeiro busca o máximo de independência desse sujeito, bem como a prevenção de novos episódios de AVC e o aparecimento de outras morbidades. Proporciona, ainda, esclarecimento do cuidador na continuidade da assistência em domicílio.

A assistência deve ser sistematizada durante o atendimento do cliente, em todas as etapas. Faz-se necessária maior discussão das atividades cotidianas, atentando-se para os aspectos subjetivos do cuidar da pessoa com deficiência.

\section{REFERÊNCIAS}

1. Bocchi SCM, Ângelo M. Interação cuidador familiarpessoa com AVC: autonomia compartilhada. Ciênc Saúde Col. 2005;10(3):729-38.

2. Rouquayrol MZ. Epidemiologia e Saúde. $6^{\mathrm{a}}$ ed. Rio de Janeiro: MEDSI; 2003.

3. Silva AMF, Heidemann ITSB. Desvelando a deficiência em busca da cidadania. Acta Paul Enferm. 2002 Jan/ Mar;15(1) 79-89.

4. Brasil. Ministério da Saúde. Resolução 37/52 de 3.12.1982. Documento das Nações Unidas. Programa de Ação Mundial para as Pessoas Deficientes- Política Nacional de Saúde da Pessoa Deficiente- Disponível em www.portal.saude.gov.br/portal/arquivos/pdf/ manual2.pdf Acessado em 12/9/2006

5. Ring H. La rehabilitación en los ancianos. In.: Organización Panamericana De La Salud. La atención de los ancianos: un desafio para los años noventa. Washington: Elias Anzola Pérez;1994.

6. Poter PA e Perry AG.Grande Tratado de enfermagem prática - conceitos básicos, teoria e prática hospitalar. $3^{a}$ ed. São Paulo: Santos; 1998.

7. Machado WCA, Figueiredo NMA, Scramin AP. Cuidar de longo prazo e reabilitação do cliente. In: Santos, I Figueiredo NMA, Padilha MICS et al. Enfermagem assistencial no ambiente hospitalar: realidade, questões, soluções. São Paulo: Atheneu; 2004. v.2, 
cap. 28, p. 445-82.

8. Diogo MJD. O papel da enfermeira na reabilitação do idoso. Rev Latino-Am Enferm. 2000 Jan;8(1):75-81.

9. Machado WCA. . O papel do enfermeiro no cuidar de clientes portadores de deficiência. Disponível em www.entreamigos.com.br

10. Rolim M, Castro M. Compliance to the Hypertension Control Program and the Standardized Nursing Results: an exploratory study. Online Brazilian Journal of Nursing [Online] 6:1. Available www.uff.br/objnursing

11. Hinds OS, Chaves DE, Cypress SM. Context as a source of meaning and understanding. Qual Health Res. Newbury Park. 1992;2(1):61-74.

12. Mantovani M, Mottin J, Rodrigues J. Nurse Home Visity with Educational Activities in the arterial pressure treatment. Online Brazilian Journal of Nursing [Online] 6:1. Available. www.uff.br/ojs-2.1.1/index.php/nursing/ rt/printerFriendly/757/171

13. Brasil. Ministério da Saúde. Secretaria de Atenção à Saúde. Departamento de Atenção Básica. Hipertensão arterial sistêmica para o Sistema Único de Saúde / Ministério da Saúde, Secretaria de Atenção à Saúde, Departamento de Atenção Básica. - Brasília : Ministério da Saúde, 2006. 58 p. - (Cadernos de Atenção Básica; 16) (Série A. Normas e Manuais Técnicos).

14. Couto RC. Emergências médicas e terapia intensiva. Rio de Janeiro: Guanabara Koogan; 2005.

15. Brasil. Ministério da Saúde. Secretaria de Atenção à Saúde. Departamento de Atenção Básica. Prevenção clínica de doenças cardiovasculares, cerebrovasculares e renais / Ministério da Saúde, Secretaria de Atenção à Saúde, Departamento de Atenção Básica. - Brasília : Ministério da Saúde, 2006. 56 p. - (Cadernos de Atenção Básica; 14) (Série A. Normas e Manuais Técnicos).

16. Yamashita LF, Fukujima MM, Granitoff N, Prado GF do. Paciente com acidente vascular cerebral isquêmico já é atendido com mais rapidez no hospital. Arq Neuropsiq. 2004;62(1):96-102.

17. Orientação Funcional para a Utilização da MIF - Medida de Independência Funcional (MIF para Adultos) Versão Brasileira. Divisão de Medicina de reabilitação do Hospital das Clínicas da Faculdade de Medicina da Universidade de São Paulo. Manual.

18. Battistella LR, Brito CMM. Classificação Internacional de Funcionalidade (CIF). Acta Fisiat. 2002;9(2):98-101.

19. Chagas NR, Monteiro ARM. Educação em saúde e família: o cuidado ao paciente, vítima de acidente vascular cerebral. Acta Scient Health Sciences. 2004. 26(1):193-204. www.ppg.uem.br/Docs/ctf/Saude

20. Perlini NMOG, Faro ACM. Cuidar de pessoa incapacitada por acidente vascular cerebral no domicílio: o fazer do cuidador familiar. Rev Esc Enferm USP. 2005;39(2):154-63.

21. Nolan J, Nolan M, Booth A. Developing the nurse's role in patient education: rehabilitation as a case example. Internat J Nurs Stud. 2001;38:163-73. Disponível em www.elsevier.com/locate/ijnurstu

22. Kummer K, Echer IC. Meaning of the participation in a support group for family members of dependent patients for the undergraduate nursing student. Online Braz J. Nurs [periódico na internet] 2005 Aug;4(2) Disponível em www.uff.br/nepae

23. Pérez RCF, Carrión MAF, Quintana JAM. Acciones de enfermería en pacientes con afecciones cerebrovasculares. Rev Cubana Enferm [periódico na internet]. 2002 Set/Dez;18(3):189-93. Disponível em www.scielo.sld.cu/scielo

24. Brasil. Ministério da Saúde Resolução da Diretoria Colegiada RDC 11 de janeiro de 2006 Dispõe sobre o Regulamento Técnico de Funcionamento de Serviços que prestam Atenção Domiciliar. Disponível em www.elegis.anvisa.gov.br/leisref/public.

25. Silva KL, Sena R, Leite JCA, Seixas CT, Gonçalves AM. Internação domiciliar no Sistema Único de Saúde. Rev. Saúde Pública [periódico na Internet]. 2005 Jun 39(3): 391-97. Disponível em: www.scielo.br/scielo.php?script

26. Ruipérez I, Llorente P. Acidente vascular cerebral: importância, causas e conseqüências; cuidados durante as diferentes fases evolutivas. Cap. 15 - Geriatria- Guias Práticos de Enfermagem 213-42. Rio de Janeiro -RJ; 2000.

27. Langhorne P. Review: early supported discharge may reduce length of hospital stay in patients with acute stroke, but does not reduce death. Cochrane Database Syst Rev 2002;(1):CD000443 www.ebnbmjjournals.com

28. Sulch D, Perez I, Melbourn A, Kalra L. Randomized Controlled Trial of Integrated (Managed) Care Pathway for Stroke Rehabilitation Evidence. Based Nursing 2001; 4:53 ㄷ 2001 Evidence-Based Nursing. 
29. Mesquita SRAM, Anselmi ML, Santos CB, Hayashida M. Programa interdisciplinar de internação domiciliar de Marília-SP: custos de recursos materiais consumidos. Rev Latino-Am Enferm. 2005 Jul/Ago;13(4):555- 61.

30. Rede Sarah de Hospitais de Reabilitação. Informação sobre doenças tratadas: Acidente Vascular Cerebral. www.sarah.br/paginas/doencas

31. Brasil. Ministério da Saúde. Sistema Único de Saúde, Lei 8.080 de 19 de setembro de 1990. Dispõe sobre as condições para a promoção, proteção e recuperação da saúde, a organização e o funcionamento dos serviços correspondentes e dá outras providências. Disponível em www.portal.saude.gov.br/portal/arquivos. Acessado em 03/09/2006.

32. Sena R, Silva K, Rates H, Vivas K, Queiroz C, Barreto F. O Cotidiano da cuidadora no domicílio: desafio de um fazer solitário. Cogitare Enferm. 2006 Mai/ Ago;11(2):124-32. 